\title{
Validation of the sino-nasal outcome test-23 in septorhinoplasty surgery*
}

\author{
Arunjit S. Takhar, Joanna Stephens, Premjit S. Randhawa, Anne-Lise Poirrier, \\ Peter Andrews
}

Department of Facial Plastics \& Rhinology, The Royal National Throat, Nose \& Ear Hospital, 330 Gray's Inn Road, London, WC1X 8DA, United Kingdom
Rhinology 52: 320-326, 2014

DOl:10.4193/Rhino14.009

*Received for publication:

January 14, 2014

Accepted: March 8, 2014

\begin{abstract}
Background: There has been a great expansion in patient-based outcome measures to face the ever-increasing demand to demonstrate surgical efficacy. However, surgeons have not adopted until now any systematic outcome instrument. The 22-item Sino-Nasal Outcome Test (SNOT-22) is a validated sinonasal outcome score in sinonasal and septorhinoplasty surgery but does not measure aesthetic outcome. The aim of this paper is to validate a modification to the Sino-Nasal Outcomes Test-22 (SNOT-22) with a new question regarding the shape of the nose (SNOT-23).
\end{abstract}

Methodology: We conducted a prospective cohort study on 69 consecutive patients undergoing septorhinoplasty and a control group of healthy volunteers. Reproducibility, responsiveness to treatment, known group differences and validity of the SNOT-23 were analysed. Scores were compared to visual analogue scales, nasal obstruction symptoms evaluation (NOSE) score and nasal inspiratory peak flow.

Results: SNOT-23 has good test-retest reliability and is a valid outcome measure for assessing response to surgery especially with regards to shape of the nose and nasal obstruction when compared to other patient reported measures.

Conclusion: SNOT-23 is a valid and reliable tool that can be easily used in routine clinical practice to assess the functional and aesthetic outcomes from septorhinoplasty surgery. The SNOT-23 could be adopted as a universal, easy to use tool in rhinology clinics for the assessment of response to septorhinoplasty and sinus surgery.

Key words: nasal reconstruction, outcome, functional septorhinoplasty, nasal obstruction, quality of life, Sino-Nasal Outcome Test-22

\section{Introduction}

The aim of functional septorhinoplasty surgery is to restore an adequate nasal airway whilst retaining or improving the aesthetic appearances of the nose. The assessment of a patient's nose can be difficult due to the subjectivity of the complaints. Whilst the surgeon can assess the deformity aesthetically, and use tools such as acoustic rhinometry and nasal inspiratory peak flow to objectively determine nasal obstruction, ultimately the patient's subjective and qualitative satisfaction will dictate the success of the surgical outcome ${ }^{(1,2)}$.

Patient reported outcome measures (PROMs) seek to ascertain patients' views of their symptoms, their functional status, and their health related quality of life and are becoming more important in clinical medicine not only for assessing benefit of an intervention, but also for commissioning of services as it provides the best approximation of the burden of a disease to the patient. They can also be used as a comparator for departmental or even individual surgeons' outcomes ${ }^{(3)}$.

A wide variety of disease specific PROMs have been developed and validated which can assess outcomes of intervention for sino-nasal disease. One of the most extensively studied tools to date is the Sino-Nasal Outcomes Test-22 (SNOT-22) which has become widely integrated into routine clinical practice when assessing and managing chronic rhinosinusitis ${ }^{(4,5)}$. The 
SNOT-22 is a disease-specific, quality of life outcome measure of sino-nasal function which has been demonstrated to be reliable, valid and responsive ${ }^{(6.7)}$. In recent studies of its use in septorhinoplasty surgery it has been shown to be a useful tool to assess outcomes ${ }^{(8-11)}$ but does not have a question specifically about the shape of the nose which may limit its usefulness as a single, specific tool for functional septorhinoplasty. A number of studies have demonstrated the benefits of using multiple tools to measure outcomes such as the nasal obstruction symptoms evaluation (NOSE), Rhinoplasty Outcomes Evaluation (ROE) and sleep outcomes survey (SOS) ${ }^{(12,13)}$ but these focus on individual functional symptoms and do not fully address psychological effects of surgery on quality of life.

We developed a modification of the SNOT-22 with a question regarding 'concern with shape of the nose' to create the Sino-Nasal Outcome Test-23 (SNOT-23: Figure 1). The goal of this study was to assess the reliability, validity, responsiveness and interpretability of the SNOT-23. We evaluated the test-retest reliability and responsiveness to septorhinoplasty at three months after surgery. We compared the score to a healthy population, and to other tools such as visual analogue scale (VAS), NOSE score and nasal inspiratory peak flow.

\section{Materials and methods}

Population

A prospective study of 69 consecutive patients undergoing functional and reconstructive septorhinoplasty surgery was conducted over a 10-month period at the Royal National Throat, Nose and Ear Hospital, London. The procedures were performed by either endonasal or external approach under general anaesthetic by one team of surgeons. The exclusion criteria were patients under the age of 16 , inability to give consent, understand the English language or the questionnaire, concomitant functional endoscopic sinus surgery or other nasal airway procedure.

To evaluate the ability of the SNOT-23 to reflect 'known group' differences, SNOT-23 scores were collected in 10 healthy volunteers randomly selected from the staff of the hospital. They were not known to be suffering with a nose or sinus condition and were consented to take part.

For all subjects, information was collected regarding the following: age, gender, surgical technique, surgeon, history of trauma, co-existing sinonasal disease, allergy, smoking status, and previous nasal surgery.

\section{Validation}

Patients completed the SNOT-23 questionnaire on two separate occasions pre-operatively (Test-Retest Reliability) and at one of

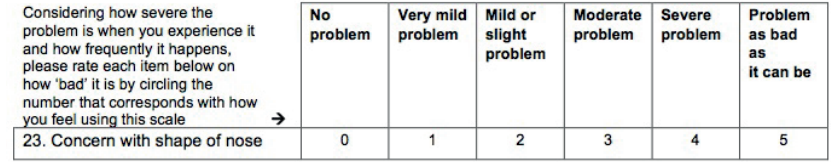

Figure 1. Sino-Nasal Outcome Test-23 (SNOT-23). Our modification to the SNOT-22 involved adding the additional question regarding shape of the nose. Symptoms are rated from 0 (no problem) to 5 (problem as bad as it can be). The theoretical range of the measure is from 1 to 115 , with a higher score indicating poorer nasal function or impact upon quality of life.

their follow-up visits at three months post-operatively (Responsiveness). They were also asked to complete NOSE, visual analogue scores and nasal inspiratory peak flow measurements at the same time. Patients were not allowed to view their previous responses when completing the questionnaires.

The SNOT-23 questionnaire rated 23 different symptoms from 0 (no problem) to 5 (problem as bad as it can be) giving a theoretical total score of between 0-115 (Figure 1). The NOSE questionnaire is a validated disease-specific, quality-of-life instrument that is used to specifically assess the component of nasal obstruction ${ }^{(14)}$. It is commonly used in otolaryngology practices to provide an objective measure of nasal obstruction. The instrument is brief and easy to complete, with minimal respondent burden. It consists of 5 questions seeking to rate the burden of nasal obstruction during the past month scored from 0 (not a problem) to 4 (severe problem) giving a score of between 0-20. It is well validated and has been used to measure quality of life improvements in nasal septal surgery, functional septorhinoplasty and nasal valve surgery with good effect ${ }^{(15-17)}$. Visual analogue scales for nasal blockage, shape of the nose and overall nasal symptoms were completed on a scale of 0-10 in terms of severity. Nasal inspiratory peak flow measurements were taken using a mini-wright peak flow meter and anaesthetic facemask; the optimal recording of three serial measures in litres/minute was used.

\section{Statistical analysis}

Test-retest reliability of the SNOT-23 was assessed by matched responses at the initial and subsequent repeat questionnaires pre-operatively. The test of normality (Shapiro-Wilk) did not show normally distributed results therefore responsiveness to septorhinoplasty at three months was evaluated using the paired Wilcoxon test. In a subgroup of 18 patients, we also recorded the post-operative score after six months. The SNOT-23 was correlated to the NOSE score, VAS scale and nasal inspiratory peak flow using Spearman regression. Comparison with the control group of 10 healthy individuals used the unpaired Mann- 
Table 1. Demographic and clinical data.

\begin{tabular}{|lc|}
\hline Demographic & Data Value \\
\hline Total number & 69 \\
\hline Age (mean \pm SD), yr & $34.5 \pm 12.9$ \\
\hline Sex, no, (\%) & \\
\hline \multicolumn{1}{|c|}{ Male } & $46(66.7)$ \\
\hline Female & $23(33.3)$ \\
\hline History \& Co-Morbidites & Data Value (\%) \\
\hline Nasal Trauma & 73.9 \\
\hline Revision Surgery & 29.0 \\
\hline Smoking & 31.9 \\
\hline Allergic Rhinitis & 15.9 \\
\hline Surgical Data & Data Value \\
\hline Surgical Technique (\%) & \\
\hline External Approach & 36.2 \\
\hline Endonasal Approach & 29.0 \\
\hline Seniority of Surgeon (\%) & 71.0 \\
\hline Consultant & \\
\hline Mean Follow-up (days) & \\
\hline
\end{tabular}

Whitney U Test.

\section{Results}

Population

Sixty-nine subjects (46 males, 23 females) were included in this study. The mean age was 34.5 years ( \pm 12.9 years). The majority of patients underwent an external approach (71\%) and the remainder (29\%) underwent an endonasal approach. Twenty patients (29\%) were revision cases, including sixteen external approach and four endonasal septorhinoplasties. Surgeries were carried out by the consultant (senior author) in $63.8 \%$ of cases and the remaining cases were performed by ear, nose and throat trainees under supervision (36.2\%). The mean time to follow-up with questionnaire assessments was 99.08 days (i.e. 14 weeks). The demographic and medical data are summarized in Table 1. Responses to all items were well distributed across response categories.

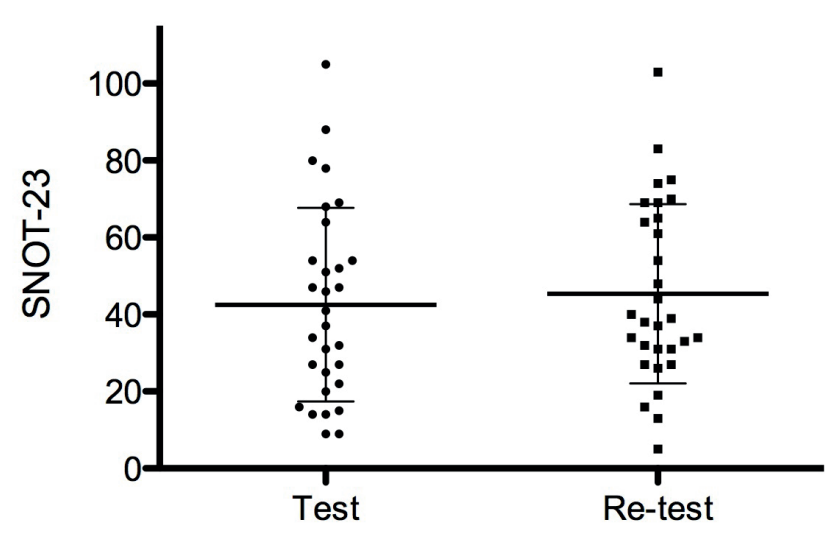

$$
\begin{aligned}
& r=0.8116 \\
& p<0.0001
\end{aligned}
$$

Figure 2. Test-Retest Reliability. The SNOT-23 was completed by patients in clinic (test) and again on the day of surgery (re-test). The figure shows the distribution of cumulative SNOT-23 scores along with mean and standard deviation distribution for both groups. $r=$ correlation coefficient.

\section{Test-Retest reliability}

The test-retest reliability measures the stability of an instrument over time with repeated testing and was assessed for SNOT-23 by administering the questionnaire to responders on two different occasions pre-operatively without subjects being able to see their previous responses. The degree of correlation between the two scores should be $\geq 0.7$ to demonstrate good reliability. When the summary scores for both pre-operative questionnaires were matched, the test-retest reliability coefficient was 0.81 indicating a good reliability (Figure 2).

We also measured the test-retest coefficient for some of the individual disease specific questions. For question twenty two relating to nasal blockage the coefficient was 0.63 whereas for the twenty third question regarding shape of the nose the coefficient was 0.78 suggesting that the question relating to shape of the nose was more reliable than blockage in septorhinoplasty patients.

\section{Post operative outcome}

The mean pre-operative score was $51.6( \pm 26.2)$ and postoperatively this was 25.7 ( \pm 22.0 ). The impact of surgery upon SNOT-23 scores was highly significant $(p<0.0001)$ indicating a good responsiveness of SNOT-23 score to septorhinoplasty. Overall pre- and post-operative scores are summarised in Figure 3. The overall post-operative score was improved in $91.3 \%$ of patients who underwent surgery. Patients with higher pre- 


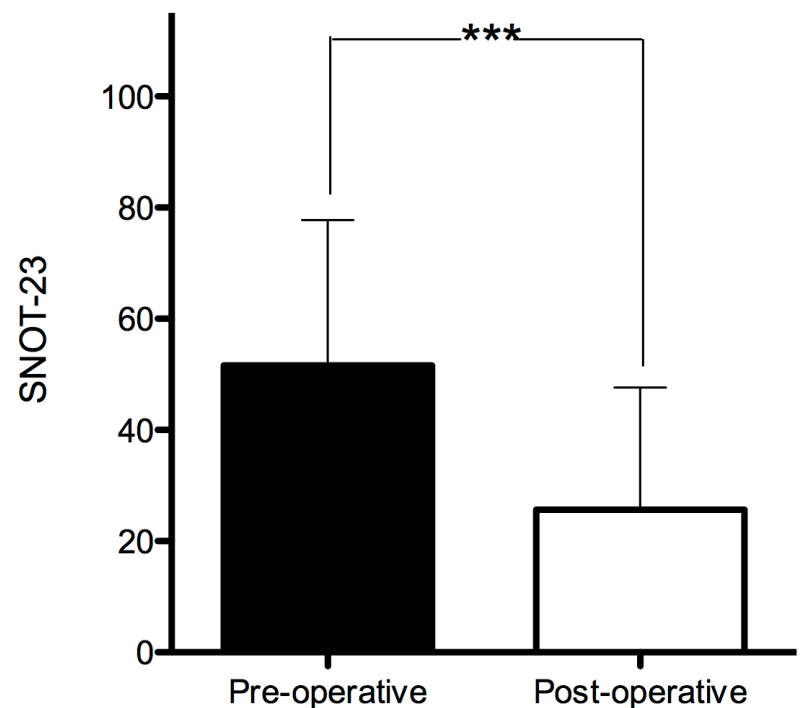

Figure 3. Post Operative Outcome. Pre-operative and post-operative SNOT-23 scores. The overall score improved significantly after septorhinoplasty $\left({ }^{* * *} \mathrm{P}<0.0001\right)$. The mean pre-operative score is shown in black and the mean post-operative score is in white. Data presented as mean \pm standard deviation.

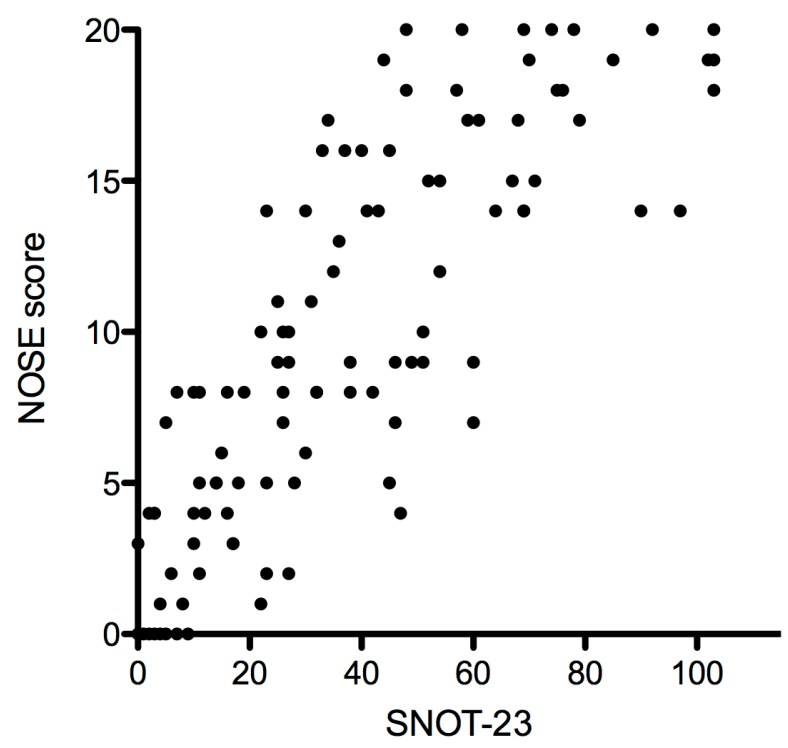

Figure 5. Correlation to Nasal Obstruction Symptoms Evaluation. Diagram shows high correlation between SNOT-23 scores ( $x$-axis) and total score for NOSE questionnaire (y-axis). (Spearman $r 2=0.8169 ; \mathrm{p}<$ 0.0001).

operative scores demonstrated the largest reduction in scores post-operatively i.e. the biggest improvement.

The responsiveness of specific questions of the SNOT-23 after surgery was also measured. The responsiveness of question twenty-two (nasal obstruction) was highly significant $(\mathrm{p}<$
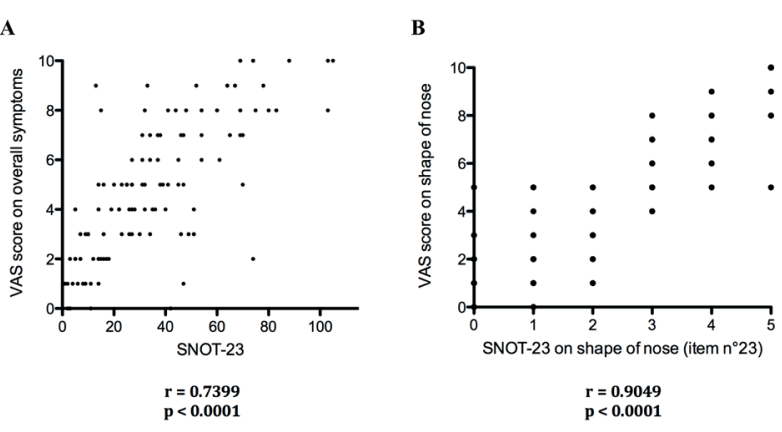

Figure 4. Correlation to visual analogue scale instrument. We found a high correlation between the SNOT-23 score and the VAS instrument another quality of life instrument. (A) Correlation between overall SNOT-23 score ( $x$-axis) and the scoring of global nose symptoms on a VAS instrument ( $y$-axis) was high (Spearman $r 2=0.7399$ ). (B) Correlation between item relating to shape of nose on SNOT-23 questionnaire (x-axis) and specific question regarding shape of nose on the VAS instrument (y-axis) was also high (Spearman $r 2=0.9049)$.

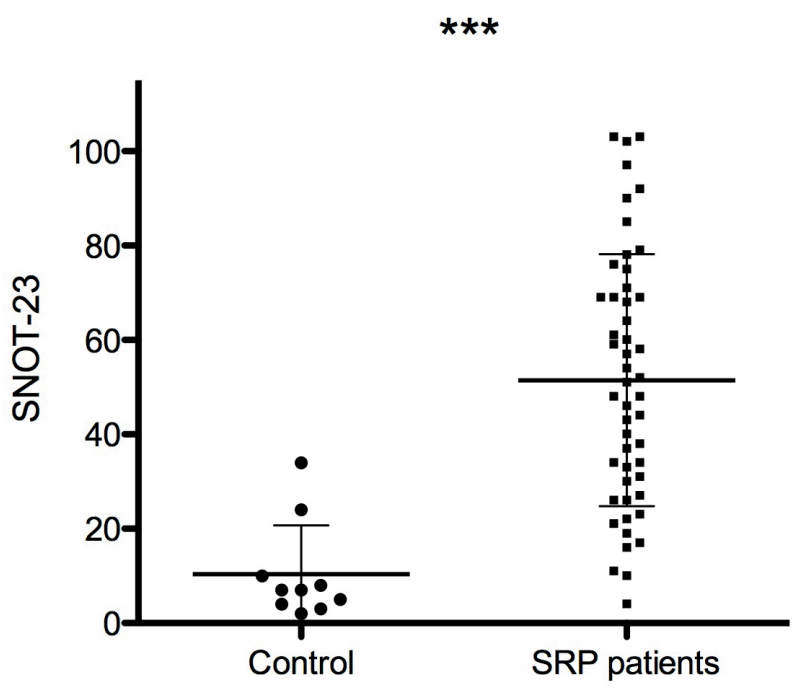

Figure 6. Comparison to a control group. The ability of the SNOT-23 to detect 'known group' differences was assessed by comparing the preoperative study group to a control group of healthy volunteers. Using the unpaired $U$ Mann-Whitney Test we found a significant difference $\left({ }^{* * *} \mathrm{p}<0.0001\right)$ between the total SNOT-23 scores. Graph shows distribution of scores, mean and standard deviation. SRP = Septorhinoplasty.

$0.0001)$ as was the twenty third question relating to the shape of the nose $(p<0.0001)$. In eighteen patients repeated post-operative scores were measured again at six months after surgery and demonstrated continued improvement in scores $(p=0.071)$. There were a total of 5 (7.25\%) complications, which were all either epistaxis or surgical site infection graded as I or II according 
to the Clavien-Dindo classification system ${ }^{(18)}$. There were no long-term sequelae recorded from any of these complications.

\section{Correlation to other patient reported measures}

The scores from the SNOT-23 were compared to corresponding visual analogue scale (0-10) and the NOSE questionnaire cumulative scores. Correlation between the overall SNOT-23 score and the scoring of the global nose symptoms on the visual analogue scale showed a high correlation between SNOT-23 and visual analogue scale responses (Spearman $\mathrm{r} 2=0.74$ ). The correlation between scores for shape of the nose on SNOT-23 and on the VAS was also high (Spearman $r 2=0.90$ ). These findings are summarized in figure four. The cumulative score for the NOSE questionnaire (out of 20) was also found to correlate highly with total SNOT-23 scores (Spearman $\mathrm{r} 2=0.82$ ) suggesting this is measuring highly related parameters (Figure 5).

\section{Comparison to nasal inspiratory peak flow}

Nasal inspiratory peak flow was our objective measure that was recorded before and after surgery. The mean pre-operative peak flow score was 87.8 ( \pm 29.6) litres /minute and post-operatively it was 99.5 ( \pm 39.6$)$ litres / mean $(p=0.0328)$. There was no correlation between improvement in nasal inspiratory peak flow readings and total SNOT-23 scores.

\section{Comparison to a control group}

The ability of SNOT-23 to reflect 'known group' differences was examined to help determine the measure's validity. The 69 preoperative SNOT-23 scores were compared to a control group of 10 healthy volunteers and the results analysed using the unpaired Mann-Whitney $U$ test.

The average age of the control group was 30.3 years ( \pm 6.3 years) compared to the study group with an average age of 34.5 years $( \pm 12.9)$. The average total SNOT-23 score in the control group was 10.4 ( \pm 10.4 ) compared to 51.6 ( \pm 26.2 ) in the pre-operative patients in the study group. There was a statistically significant difference $(p<0.0001)$ between the control group suggesting the SNOT-23 is a valid measure (Figure 6).

\section{Discussion}

This study evaluated 69 patients undergoing functional septorhinoplasty and demonstrated the feasibility and reliability of using the SNOT-23 in routine clinical practice. The SNOT-22 has already been shown to be a useful measure in septorhinoplasty to assess response to surgery with regards to generic quality of life measures and nasal obstruction ${ }^{(10)}$. This study now shows that adding a question regarding the shape of the nose is valid reliable and responsive to surgery. Total SNOT-23 scores were consistent with NOSE and VAS scores. Improvements noted in the shape of the nose were consistent with a visual analogue scale of the same symptom.

Patients were screened for co-morbid conditions. Our series included a high rate of traumatic (73.9\%) and revision cases (29\%), which is consistent with data from similar studies ${ }^{(12)}$. The rate of allergic rhinitis was $15.9 \%$ in our series which is slightly lower than the rate in the general population where it is considered to be between $19-23 \%{ }^{(19)}$. This may falsely improve our results, although it should be noted that in normal circumstances allergic rhinitis should be fully treated prior to considering a patient for septorhinoplasty. The rate of smokers (31.9\%) was considerably higher than in the general population according to current estimates ${ }^{(20)}$.

There were a total of six patients who failed to demonstrate an improvement in their total SNOT-23 scores at three months post-operatively. Amongst these, four of the patients had subsequent six month follow-up scores available which were equivalent or better than their pre-operative score. These patients all scored lower on the blockage and shape domains of the instrument at six months post-operatively suggesting other nondisease specific reductions in quality of life were influencing their scores. In the remaining two patients the post-operative scores were only taken at six and eight weeks and they have yet to attend further long-term follow-up. Although post-operative scores were ideally taken at three months after septorhinoplasty, the nature of clinic appointment scheduling dictated that some patients were seen at less than three months. In theses cases the on going healing process and resolution of inflammation may still have a negative impact on the true assessment of longerterm surgical outcome measurement when using this tool.

The objective measurement of nasal airway patency used in this study was nasal inspiratory peak flow. We found general improvements in readings post operatively, which is consistent with other similar studies ${ }^{(8,17)}$ suggesting overall improvement in nasal obstruction. However these studies do not correlate improvement of objective scores with improvement in quality of life measures, when this was performed in our study we actually found no correlation. Objective measures of nasal patency (nasal peak flow, rhinomanometry, acoustic rhinometry) have limitations in the clinical setting ${ }^{(21,22)}$ and although they provide an objective measure they reflect only one aspect of the disease. Our study provides further evidence that they do not encompass other aspects of the disease, and therefore may be of limited overall clinical value as a measure of outcome. Other problems with such objective outcome measures include cost, maintenance of equipment and appropriate staff training. Questionnaires are a simple and cheap method that can assist the surgeon in selecting patients for surgery and providing reliable follow-up with good compliance. They are repeatable 
and the numerical representation of the test results allows for easy visualisation and comparison of outcomes after treatment. Being disease specific, the SNOT 23 allows the most important aspects of the disease that affects the patient to be recorded which can subsequently help the surgeon to best meet the patient's expectations ${ }^{(2)}$.

In recent years, there has been a great expansion in the number of instruments used to measure patient reported outcomes. They are likely to play an increasingly important measure of quality of healthcare services and may form part of commissioning of services in rationed healthcare systems such as in the UK ${ }^{(3)}$. The nasal obstruction symptom effectiveness score (NOSE) and the rhinoplasty outcomes evaluation (ROE) score have been applied to assess outcomes in functional rhinoplasty $(12,13,23,24)$ but these tools do not fully evaluate general health, emotional or psychological aspects. SNOT-22 has been widely adopted in rhinosinusitis and sinus surgery ${ }^{(6,7,25)}$ and is widely regarded as the most useful sinonasal outcome scoring system ${ }^{(4,5)}$. Its validity has been demonstrated in septal surgery ${ }^{(26,27)}$ and a number of recent studies have demonstrated its responsiveness to septorhinoplasty ${ }^{(8,10,17)}$.

Despite the evidence available to date and the increasing influence of patient reported outcome measures there has not been a widespread adoption of a single, reliable instrument to assess and follow-up patients undergoing septorhinoplasty surgery. This may be partially attributable to the lack of specificity to septorhinoplasty surgery that is conferred by the SNOT-22 as it only addresses disease specific symptoms of nasal obstruction and not the shape of the nose. Our modification to create the SNOT-23 provides a single, specific, reliable and easy to perform instrument that could become invaluable in septorhinoplasty surgery to fully assess outcome. We acknowledge that the postoperative outcomes may be attributable to nonsurgical factors such as the natural course of the disease or psychological issues (28)

In order to evidence subjective measures of nasal symptoms in the outpatients clinic there is currently a requirement to use a variety of tools within one clinic such as the rhinoplasty outcomes evaluation and SNOT-22. Whilst some of the symptoms evaluated by the questionnaire appear to be of little relevance to our patient group (cough, ear fullness and dizziness) other questions such as embarrassed, irritable and nasal blockage have been shown to improve after surgery ${ }^{(10)}$. The addition of a question regarding the shape of the nose has also been shown by this study to improve considerably after surgery. The suggestion is that by encompassing a large range of symptoms they cover other feelings contributing towards aspects of the nasal problem. Given the widespread adoption of the SNOT-22 to routine clinical practice for rhinosinusitis, the SNOT-23 could become a single, easy to use instrument in the outpatients setting to help manage all rhinology patients without the need for the multiple, disease specific and generic questionnaires that are currently available for different rhinology conditions. It has been shown to be reliable, valid and responsive to treatment.

\section{Conclusion}

Overall the SNOT-23 score improved significantly in patients undergoing septorhinoplasty surgery and the addition of a question regarding the shape of the nose has been shown to be clinically relevant, valid and reliable. The tool also showed good correlation with other disease specific outcome measures. Further evaluation across more surgical centres is required to fully validate the tool and determine what exactly confers outcome. Provided that the twenty-third item does not affect validity in rhinosinusitis patients, SNOT-23 could become the universal rhinology tool. The score is easy to perform, interpret and implement in routine clinical practice, and adds a subjective yet valid and reliable dimension to the objective measurements.

\section{Authorship contribution}

All authors were members of the RNTNE rhinology \& facial plastic team and contributed to the care of the patients. ALP and PA designed the study, and together with AT, PR and JS collected the data. AT, PR and JS were involved in literature search. PA, ALP, $\mathrm{PR}$ and JS conducted all surgical procedures. Data interpretation was performed by ALP. Writing was performed by AT and PA.

\section{Conflicts of Interest}

No conflicts of interest.

\section{Acknowledgement}

Licensing and copyright permissions for the modification of SNOT-22 have been applied for to Washington University in St Louis, Missouri, USA. Thanks to Prof J Piccirillo for his support.

\section{References}

1. Yu K, Kim A, Pearlman S. Functional and aesthetic concerns of patients seeking revision rhinoplasty. Arch Facial Plast Surg 2010; 120: 291-297.

2. Steiger J. The rhinoplasty consult. Facial Plast Surg 2011; 5: 393-396.
3. Black N. Patient reported outcome measures could help transform healthcare. BM 2013; 346 (f146).

4. Hopkins C. Patient reported outcome measures in rhinology. Rhinology 2009; 47: 10-17.

5. Morley AD, Sharp HR. A review of sinonasal outcome scoring systems - which is best?
Clinical Otolaryngology 2006; 31: 103-109.

6. Hopkins C, Gillett S, Slack R, Lund V, Browne J. Psychometric validity of the 22-item Sinonasal Outcome Test. Clinical Otolaryngol 2009; 34: 447-454.

7. Erekosima NU, Katial RK. Assessing nasal symptom control. Curr Allergy Asthma Rep 
2009; 9: 134-139.

8. Timperley D, Stow N, Srubiski A, Harvey R, Marcells G. Functional outcomes of struc tured nasal tip refinement. Arch Facial Plast Surg 2010; 12: 298-304.

9. Poirrier A, Ahluwalia S, Kwame I, Hannan S, Bentley M, Andrews P. External Nasal Valve Collapse: Surgical Techniques and Outcome Measurement. Rhinology. 2014; 52: 127-132.

10. Poirrier A, Ahluwalia S, Goodson A, Ellis M, Bentley M, Andrews P. Is the Sino-Nasal Outcome Test-22 a suitable evaluation for septorhinoplasty? Laryngoscope 2013; 123 76-81.

11. Rhee JS, Poetker DM, Smith TL, Bustillo A, Burzynski M, Davis RE. Nasal Valve Surgery Improves Disease-Specific Quality of Life. Laryngoscope 2005; 115: 437-440.

12. Lindsay RW. Disease-specific quality of life outcomes in functional rhinoplasty. Laryngoscope 2012; 122: 1480-1488.

13. Saleh AM, Younes A, Friedman O. Cosmetics and function: Quality-of-Life changes after rhinoplasty surgery. Laryngoscope 2012; 122: 254-259.

14. Stewart M, Witsell D, Smith T, Weaver E, Yueh B, Hannley M. Development and validation of the Nasal Obstruction Symptom Evaluation (NOSE) scale. Otolaryngol Head Neck Surg 2004; 130: 157-163.

15. Dolan R. Minimally invasive nasal valve repair: An evaluation using the NOSE scale. Arch Otolaryngol Head Neck Surg 2010; 136: 292-295.

16. Mondina M, Marro M, Maurice S, Stoll D, de Gabory L. Assessment of nasal septoplasty using NOSE and RhinoQoL questionnaires. Eur Arch Otorhinolaryngol 2012; 269: 2189
2195.

17. Phillips P, Stow N, Timperley D, Sacks R, Srubiski A, Harvey $R$, et al. Functional and cosmetic outcomes of external approach septoplasty. Am J Rhinol Allergy 2011; 25: 351-357.

18. Clavien P, Barkun J, de Oliveira M, Vauthey J, Dindo D, Schulick R, et al. The Clavien-Dindo Classification of Surgical Complications: Five-Year Experience. Ann Surg 2009; 250: 187-196.

19. Bauchau V, Durham S. Prevalence and rate of diagnosis of allergic rhinitis in Europe. Eur Respir J 2004; 24: 758-764.

20. Office for National Statistics. A report on the 2011 General Lifestyle Survey. 2013; Available at: http://www.ons.gov.uk/ons/ rel/ghs/general-lifestyle-survey/2011/rptchapter-1.html. Accessed October, 10, 2013.

21. Eccles R. A guide to practical aspects of measurement of human nasal airflow by rhinomanometry. Rhinology 2011; 49: 2-10.

22. Nathan R, Eccles R, Howarth $P$, Steinsvåg $S$, Togias A. Objective monitoring of nasal patency and nasal physiology in rhinitis. J Allergy Clin Immunol 2005; (3 (Suppl 1)): S442-S459.

23. Yoo S, Most S. Nasal airway preservation using the autospreader technique: analysis of outcomes using a disease-specific quality-of-life instrument. Arch Facial Plast Surg 2011; 13: 231-233.

24. Most S. Analysis of Outcomes After Functional Rhinoplasty Using a DiseaseSpecific Quality-of-Life Instrument. Arch Facial Plast Surg 2006; 8: 306-309.

25. Piccirillo JF, Merritt Jr MG, Richards ML. Psychometric and clinimetric validity of the
20-Item Sino-Nasal Outcome Test (SNOT20). Otolaryngol Head Neck Surg 2002; 126: 41-47.

26. Buckland JR, Thomas S, Harries PG. Can the Sino-nasal Outcome Test (SNOT-22) be used as a reliable outcome measure for successful septal surgery? Clin Otolaryngol Allied Sci 2003; 28: 43-47.

27. Hytönen M, Lilja M, Mäkitie A, Sintonen $H$, Roine R. Does septoplasty enhance the quality of life in patients? Eur Arch Otorhinolaryngol 2012; 269: 2497-2503.

28. Tasman A. The psychological aspects of rhinoplasty. Curr Opin Otolaryngol Head Neck Surg 2010; 18(4): 290-294.

\section{Mr A S Takhar}

Department of Facial Plastics \&

Rhinology

The Royal National Throat, Nose \& Ear

Hospital

330 Grays Inn Road

London

WC1X 8DA

United Kingdom

Tel: +44-7515-915102

E-mail: aruntakhar@doctors.org.uk 